\title{
Hypertension and Its Associated Risk Factors in the Kingdom of Saudi Arabia, 2013: A National Survey
}

\author{
Charbel El Bcheraoui, ${ }^{1}$ Ziad A. Memish, ${ }^{2}$ Marwa Tuffaha, ${ }^{1}$ Farah Daoud, ${ }^{1}$ \\ Margaret Robinson, ${ }^{1}$ Sara Jaber, ${ }^{1}$ Sarah Mikhitarian, ${ }^{1}$ Mohammad Al Saeedi, ${ }^{2}$ \\ Mohammad A. AlMazroa, ${ }^{2}$ Ali H. Mokdad, ${ }^{1}$ and Abdullah A. Al Rabeeah ${ }^{2}$ \\ ${ }^{1}$ Institute for Health Metrics and Evaluation, University of Washington, 2301 Fifth Avenue, Suite 600, Seattle, WA 98121, USA \\ ${ }^{2}$ Ministry of Health of the Kingdom of Saudi Arabia, Assadah, Al Murabba, Riyadh 12613, Saudi Arabia
}

Correspondence should be addressed to Ziad A. Memish; zmemish@yahoo.com

Received 24 April 2014; Revised 2 July 2014; Accepted 2 July 2014; Published 6 August 2014

Academic Editor: Tomohiro Katsuya

Copyright (C) 2014 Charbel El Bcheraoui et al. This is an open access article distributed under the Creative Commons Attribution License, which permits unrestricted use, distribution, and reproduction in any medium, provided the original work is properly cited.

\begin{abstract}
Current data on hypertension in the Kingdom of Saudi Arabia are lacking. We conducted a national survey to inform decisionmakers on the current magnitude of the epidemic. We measured systolic and diastolic blood pressure of 10,735 Saudis aged 15 years or older and interviewed them through a national multistage survey. We used multivariate logistic regressions to describe sociodemographic characteristics and risk factors of hypertensive, borderline hypertensive, and undiagnosed hypertensive Saudis. We found that $15.2 \%$ and $40.6 \%$ of Saudis were hypertensive or borderline hypertensive, respectively. Risk of hypertension increased among men, with age, obesity, diabetes, and hypercholesterolemia. $57.8 \%$ of hypertensive Saudis were undiagnosed. These were more likely to be male, older, and diagnosed with diabetes. Among participants diagnosed with hypertension, 78.9\% reported taking medication for their condition. About $45 \%$ of participants on medication for hypertension had their blood pressure controlled. The prevalence of hypertension and borderline hypertension is very high in Saudi Arabia. Moreover, control of hypertension is poor. With the majority of hypertensive Saudis being unaware of their condition, a national plan is needed to increase utilization of freely available screening, preventive, and medical services.
\end{abstract}

\section{Introduction}

Hypertension is a leading risk factor for morbidity and mortality [1]. Untreated hypertension may lead to many serious health conditions, including stroke, aneurysms, hypertensive heart disease, coronary artery disease, kidney disease, or peripheral artery disease [2-4]. Hypertension has a major economic impact ranging from medical costs to human capital loss and decrease in productivity $[5,6]$.

The Global Burden of Disease 2010 (GBD 2010) study estimated that hypertension was the leading risk factor for death in the Kingdom of Saudi Arabia (KSA) [7]. Hypertension accounted for about $24 \%$ of total deaths from cardiovascular and circulatory diseases and $1.87 \%$ of total deaths from hypertensive urogenital, blood, and endocrine diseases [8]. From 1990 to 2010 the burden of hypertension remained very high in KSA [7]. Previous studies reported high levels of blood pressure in KSA. These levels ranged from $26.1 \%$ among individuals 30-70 years old in 1995-2000 [9] to 25.5\% among individuals 15-64 years old in 2005 [10, 11].

In order to assess the current status of hypertension in KSA, we conducted a large national survey. We selected a national sample to be representative of each of the 20 KSA health regions and the kingdom. We used an adapted standard questionnaire and took physical measurements and blood samples to examine blood pressure and chronic diseases. We used computer assisted personal interviewing to conduct the survey. The software used for offline data collection allowed interviewers to upload data to our servers on daily basis and hence a rigid monitoring of data quality.

\section{Materials and Methods}

The Saudi Health Information Survey (SHIS) is a national multistage survey of individuals aged 15 years or older. 
Households were randomly selected from a national sampling frame maintained and updated by the Census Bureau. KSA was divided into 13 regions. Each region was divided into subregions and blocks. All regions were included, and a probability proportional to size was used to randomly select subregions and blocks. Households were randomly selected from each block. A roster of household members was conducted and an adult aged 15 or older was randomly selected to be surveyed. Weight, height, and blood pressure were measured at the household by a trained professional. Omron HN286 (SN: 201207-03163F) and Omron M6 Comfort (HEM-7223-E) were used to measure weight and blood pressure.

The survey included questions on sociodemographic characteristics, tobacco consumption, diet, physical activity, health care utilization, different health-related behaviors, and self-reported chronic conditions.

We used measured weight and height to calculate body mass index (BMI) as weight $(\mathrm{kg}) /$ height $\left(\mathrm{m}^{2}\right)$. Participants were classified into four groups: (1) underweight: $\mathrm{BMI}<18.5$; (2) normal weight: BMI within 18.5-25.0; (3) overweight: BMI within 25.0-30.0; or (4) obese: BMI was greater than or equal to 30.0. Respondents were considered to be current smokers if they reported ever smoking any tobacco products and still currently smoke tobacco and past smokers if they reported smoking in the past but not anymore. We computed the servings of fruits and vegetables and red meats and chicken consumed per day from the detailed dietary questionnaire as the sum of the average daily consumption of fruits, fruit juices, and vegetables and red meats and chicken. We used the International Physical Activity questionnaire [12] to classify respondents into four groups of physical activity: (1) met vigorous physical activity, (2) met moderate physical activity, (3) insufficient physical activity to meet vigorous or moderate levels, and (4) no physical activity.

To assess diagnosed hypertension, diabetes, and hypercholesterolemia status, respondents were asked three separate questions: "Have you ever been told by a doctor, nurse, or other health professional that you had: (1) high blood pressure, otherwise known as hypertension; (2) diabetes mellitus, otherwise known as diabetes, sugar diabetes, high blood glucose, or high blood sugar; (3) hypercholesterolemia, otherwise known as high or abnormal blood cholesterol?" Women diagnosed with diabetes or hypertension during pregnancy were counted as not having these conditions. Those who were diagnosed with either of these conditions were further asked if they are currently receiving any treatment for their condition. Similarly, the same type of questions was used to determine previous diagnosis of stroke, myocardial infarction, atrial fibrillation, cardiac arrest, congestive heart failure, chronic obstructive pulmonary disease, asthma, renal failure, and cancer. We considered a person to be diagnosed with a chronic condition if they reported being diagnosed with any of the conditions cited earlier.

A total of three blood measurements were taken with the participant resting and at five-minute intervals. We followed the National Health and Nutrition Examination Survey (NHANES) for determining blood pressure levels [13].

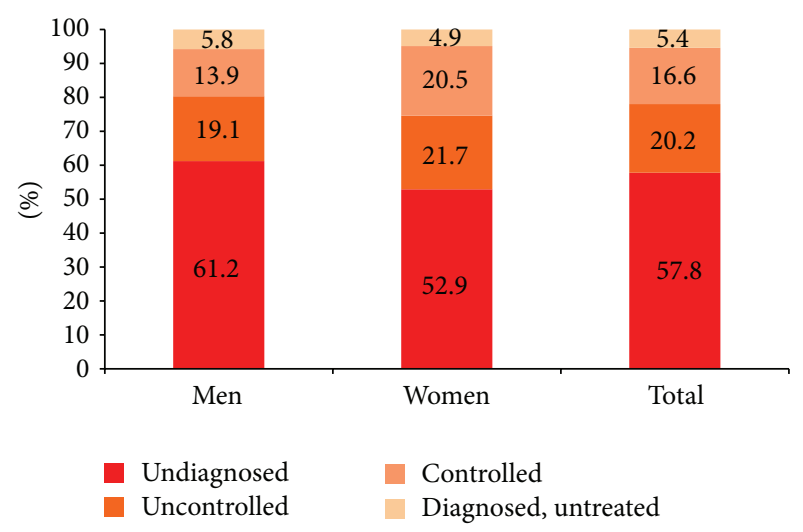

FIgURE 1: Percent distribution of diagnosis and treatment status among hypertensive Saudis aged 15 years or older, 2013.

Basically, respondents were considered to have hypertension if they met any of the following criteria: (1) measured diastolic or systolic blood pressure exceeding 89 or $139 \mathrm{mmHg}$, respectively, or (2) measured diastolic or systolic blood pressure not exceeding the appropriate threshold, but the respondent reported taking medications for hypertension. Hence, respondents who were on drugs for hypertension were considered hypertensive even if their measured diastolic or systolic blood pressure did not exceed 89 or $139 \mathrm{mmHg}$, respectively. Respondents were considered to have borderline hypertension if (1) they did not report taking drugs for hypertension and (2) their measured diastolic blood pressure was between 80 and less than $90 \mathrm{mmHg}$ or systolic blood pressure was between 120 and $139 \mathrm{mmHg}$.

We used SAS 9.2 (SAS Institute Inc., Cary, NC, USA) for analyses and to account for the complex sampling design.

\section{Results}

Between April and June 2013, a total of 12,000 households were contacted and a total of 10,735 participants completed the survey (response rate of $89.4 \%$ ). The characteristics of respondents who completed the questionnaire are presented in Table 1.

Overall, 917,188 (7.1\%) Saudis reported a diagnosis of hypertension. A total of 1,957,191 (15.2\%) Saudis aged 15 years or older had hypertension (measured or reported taking blood pressure medication). Of these, 1,119,027 were undiagnosed. Moreover, $40.6 \%$ of Saudis, or 5,222,051, had borderline hypertension. Characteristics of respondents with undiagnosed hypertension, hypertension, and borderline hypertension are presented in Table 2.

Among participants diagnosed with hypertension, $78.9 \%$ reported taking medication for their condition. About $45 \%$ of participants on medication for hypertension had their blood pressure controlled. Hence, about 390,338 adults had uncontrolled blood pressure. Among all those who are hypertensive, $57.8 \%, 20.2 \%, 16.6 \%$, and $5.4 \%$ are undiagnosed, treated uncontrolled, treated controlled, and untreated, respectively (Figure 1). 
TABLE 1: Sociodemographic characteristics, in Saudi Arabia, of males and females age 15 years or older, 2013.

\begin{tabular}{|c|c|c|c|c|c|c|c|}
\hline \multirow{2}{*}{ Sociodemographic and risk factors } & \multirow{2}{*}{ Categories } & \multicolumn{3}{|c|}{ Males } & \multicolumn{3}{|c|}{ Females } \\
\hline & & $N$ & Weighted \% & SE & $N$ & Weighted \% & $\mathrm{SE}$ \\
\hline \multirow{6}{*}{ Age (years) } & $15-24$ & 1189 & 40.79 & 1.03 & 1193 & 39.81 & 1.03 \\
\hline & $25-34$ & 1254 & 21.71 & 0.75 & 1503 & 21.28 & 0.71 \\
\hline & $35-44$ & 1132 & 13.59 & 0.53 & 1207 & 16.80 & 0.62 \\
\hline & $45-54$ & 722 & 11.97 & 0.55 & 798 & 12.80 & 0.57 \\
\hline & $55-64$ & 439 & 7.07 & 0.44 & 423 & 5.85 & 0.39 \\
\hline & $65+$ & 517 & 4.87 & 0.29 & 358 & 3.46 & 0.26 \\
\hline \multirow{3}{*}{ Marital status } & Currently married & 3514 & 49.31 & 0.98 & 3462 & 49.40 & 0.97 \\
\hline & Never married & 1569 & 49.26 & 0.99 & 1260 & 42.38 & 1.03 \\
\hline & Separated, divorced, or widowed & 159 & 1.43 & 0.15 & 738 & 8.22 & 0.43 \\
\hline \multirow{3}{*}{ Education } & Primary school or less & 1217 & 20.26 & 0.75 & 2069 & 32.52 & 0.88 \\
\hline & Elementary or high school completed & 2745 & 59.34 & 0.94 & 2127 & 46.17 & 0.98 \\
\hline & College degree or higher education & 1282 & 20.40 & 0.72 & 1275 & 21.31 & 0.77 \\
\hline
\end{tabular}

SE: standard error.

Age, sex, and diagnosis history of diabetes and hypercholesterolemia were associated with hypertension (Table 3). The risk of being hypertensive was lower among females (AOR $=0.61 ; 95 \%$ CI: $0.50-0.74$ ) but increased with age (AOR $=1.07 ; 95 \%$ CI: 1.06-1.08), among obese participants (AOR $=$ 2.24; 95\% CI: $1.89-2.65$ ) and those who have been previously diagnosed with diabetes (AOR $=1.95 ; 95 \%$ CI: $1.57-2.43$ ) and hypercholesterolemia (AOR $=1.94 ; 95 \% \mathrm{CI}: 1.51-2.47)$. On the other hand, marital status, education, smoking status, diet, time spent watching TV, levels of physical activity, diagnosis history of prediabetes, or other chronic conditions were not associated with the risk of hypertension (Table 3). Being male, older, and obese and having a diagnostic history of diabetes also increased the risk of borderline hypertension. Daily consumption of two to three servings of red meats and chicken and being moderately active was associated with the risk of borderline hypertension (Table 4).

A large percentage of hypertension was undiagnosed, as $57.8 \%$ of those with hypertension did not know of their condition, a total of 1,119,027 Saudis. The likelihood of being undiagnosed decreased among women ( $\mathrm{AOR}=0.54 ; 95 \% \mathrm{CI}$ : $0.44-0.67)$ and increased with age (AOR $=1.05$; 95\% CI: 1.05$1.06)$ and diagnosis history of diabetes $(\mathrm{AOR}=1.46 ; 95 \% \mathrm{CI}$ : 1.09-1.94) (Table 5).

\section{Discussion}

Our study revealed high rates of hypertension and borderline hypertension in KSA. Moreover, our findings revealed high rates of uncontrolled hypertension in KSA. Our findings are striking in a country with free medical care and high resources. Indeed, these findings call for action to control the burden of hypertension in the kingdom. A national plan to increase awareness, early detection, and control of hypertension is urgently needed.

Very few studies previously reported on hypertension in KSA. The most recent estimates date back to 2005 and provided a prevalence of $11.5 \%$ of reportedly diagnosed hypertension, among individuals aged 15-64 years [10], a much higher prevalence than 5.6\% from our study. Data from $1995-$ 2000 for Saudis aged 30 years or older showed a hypertension prevalence of $26.1 \%$ [9]. In comparison, we found that $27.2 \%$ of those aged 30 or older had hypertension. There are several factors that could explain these differences. First, our study is national and applied standardized methodology for data collection. Second, we used weighted analyses to generalize our findings. However, the 2005 STEPS survey and our study should be comparable and possibly indicate a leveling of the hypertension prevalence in KSA.

Our results for increased risk of hypertension with age and among men are similar to previous studies from KSA and other countries. However, literature on marital status and hypertension is inconclusive and mostly focused on comparing currently married to never married persons $[14,15]$. Our study did not show any association between marital status and high blood pressure after adjusting for confounders. This is contrary to what [9] had been reported previously on this association.

Interestingly, hypertension among Saudis did not vary with educational levels. Previous reports on association between education and hypertension are mixed as some studies have reported the lack of association [16] or an inverse relationship $[17,18]$.

While the findings on hypertension might be pointing toward stabilization or decline in prevalence, the KSA health system still has many challenges. For instance, the majority $(57.8 \%)$ of hypertensive Saudis are undiagnosed. The other $55.0 \%$ of those on treatment were not controlled. SHIS included questions on health care utilization, and only $14.8 \%$ reported visiting a health clinic for a regular checkup within the last year. It is probable that Saudis are not engaged in preventive health care and only seek medical care for illnesses. Understanding the barriers to seeking care is crucial in order to improve the health of Saudis.

Our study has some limitations. First, our data are crosssectional, and hence we cannot assess causality. Second, many of our behavioral data, such as diet and physical activity, are self-reported and subject to recall and social desirability 


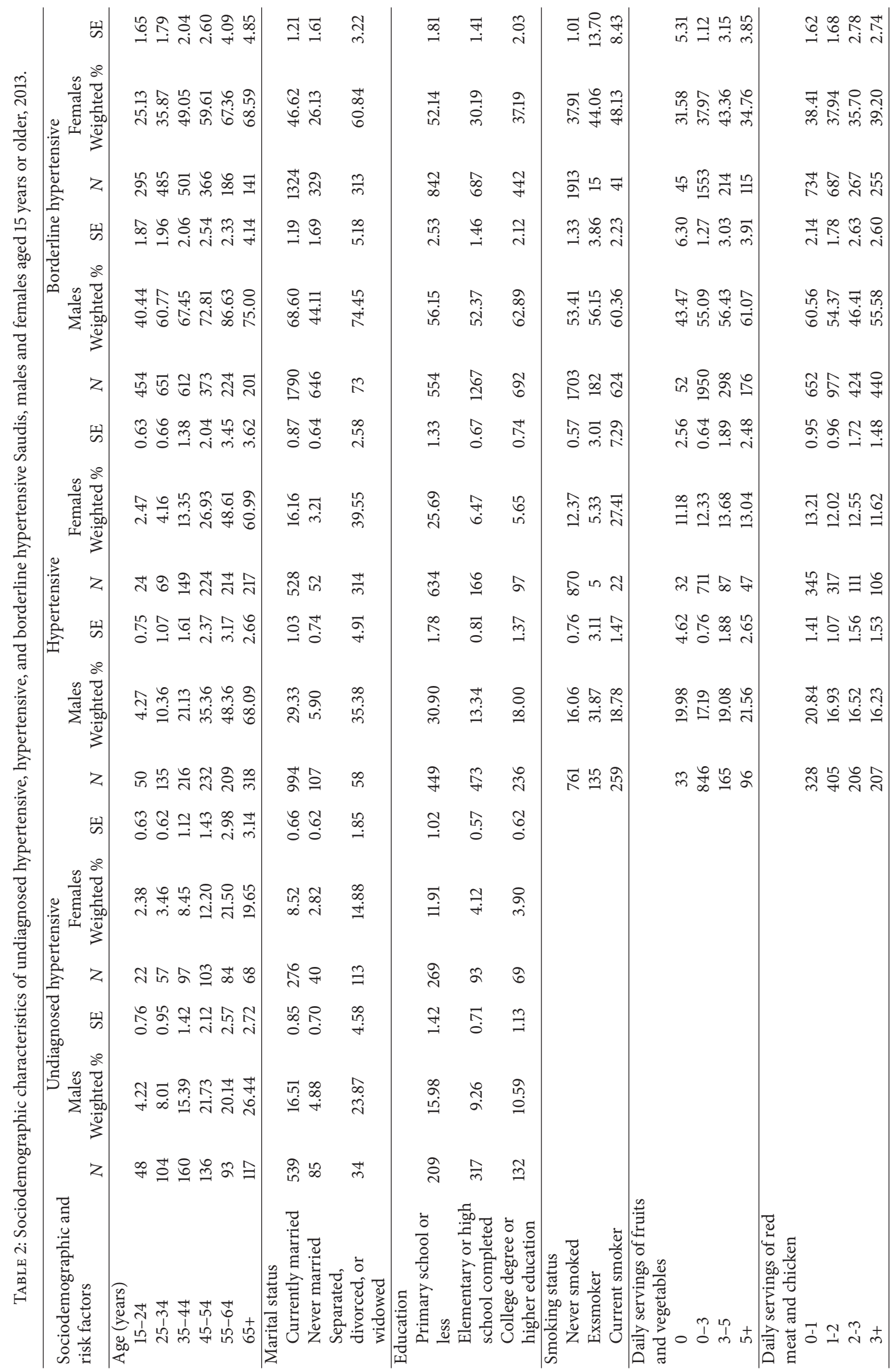




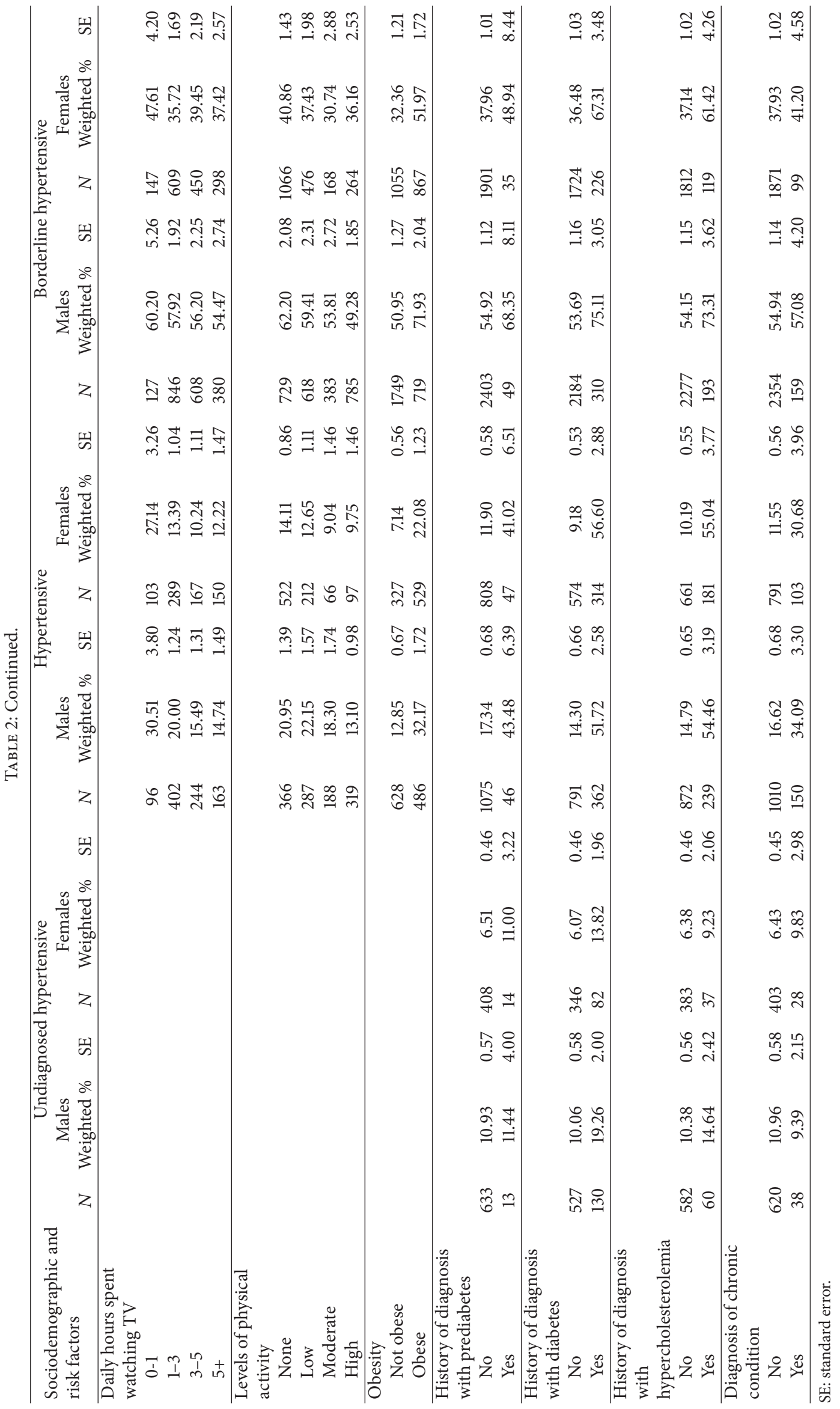


TABLE 3: Multivariate logistic regression for sociodemographic characteristics, risk factors, and hypertension, in Saudi Arabia, of males and females aged 15 years or older, 2013.

\begin{tabular}{|c|c|c|c|c|c|}
\hline \multirow{2}{*}{ Socio-demographic and risk factors } & \multirow{2}{*}{ Categories } & \multicolumn{2}{|c|}{ Sociodemographic model } & \multicolumn{2}{|c|}{ Full adjusted model } \\
\hline & & AOR & $95 \% \mathrm{CI}$ & AOR & $95 \% \mathrm{CI}$ \\
\hline \multirow{2}{*}{ Sex } & Males & REF & & REF & \\
\hline & Females & 0.61 & $0.52-0.72$ & 0.61 & $0.50-0.74$ \\
\hline Age* $^{*}$ & & 1.08 & $1.07-1.09$ & 1.07 & $1.06-1.08$ \\
\hline \multirow{3}{*}{ Marital status } & Currently married & REF & & REF & \\
\hline & Never married & 0.86 & $0.66-1.12$ & 1.05 & $0.79-1.39$ \\
\hline & Separated, divorced, or widowed & 1.29 & $1.00-1.66$ & 1.28 & $0.98-1.68$ \\
\hline \multirow{3}{*}{ Education } & Primary school or less & REF & & & \\
\hline & Elementary or high school completed & 1.01 & $0.82-1.24$ & & \\
\hline & College degree or higher education & 0.86 & $0.69-1.07$ & & \\
\hline \multirow{3}{*}{ Smoking status } & Never smoked & & & REF & \\
\hline & Exsmoker & & & 1.26 & $0.83-1.93$ \\
\hline & Current smoker & & & 1.21 & $0.95-1.55$ \\
\hline \multirow{4}{*}{ Levels of physical activity } & None & & & REF & \\
\hline & Low & & & 1.19 & $0.97-1.46$ \\
\hline & Moderate & & & 1.09 & $0.83-1.42$ \\
\hline & High & & & 1.07 & $0.84-1.35$ \\
\hline \multirow{2}{*}{ Obesity } & Not obese & & & REF & \\
\hline & Obese & & & 2.24 & $1.89-2.65$ \\
\hline \multirow{2}{*}{ History of diagnosis with diabetes } & No & & & REF & \\
\hline & Yes & & & 1.95 & $1.57-2.43$ \\
\hline \multirow{2}{*}{$\begin{array}{l}\text { History of diagnosis with } \\
\text { hypercholesterolemia }\end{array}$} & No & & & $\mathrm{REF}$ & \\
\hline & Yes & & & 1.94 & $1.51-2.47$ \\
\hline \multirow{2}{*}{ Diagnosis of chronic condition } & No & & & REF & \\
\hline & Yes & & & 1.28 & $0.93-1.76$ \\
\hline
\end{tabular}

* AOR for age should be considered as for an increase of one year.

Odds ratios were adjusted for sociodemographic characteristics: sex, age, marital status, and education.

AOR: adjusted odds ratios; CI: confidence intervals; REF: reference.

biases. On the other hand, our study is based on a large sample size and used a standardized methodology for all its measures.

Despite these limitations, our study remains nationally representative and has the merit of providing accurate data due to our near-real-time data quality monitoring through the whole survey period. The physical and blood measurements allowed us to control recall bias regarding diagnosed diseases and to uncover respondents who were affected by these chronic diseases but undiagnosed.

\section{Conclusions}

Our findings from this study along with those we reported previously in GBD 2010 [7] call for a national plan to prevent and control the burden of hypertension in KSA. Indeed, the plan has to be comprehensive to include programs to improve health behaviors such as diet and physical activity. Moreover, the plan should increase health care utilization for preventive services. As uncontrolled blood pressure leads to catastrophic events such as stroke, heart attack, and renal failure [2], physicians in KSA should be encouraged to monitor their patients to ensure that their blood pressure is controlled. Saudis should be encouraged to monitor their own blood pressure and seek medical care to control their conditions.

KSA has a young population with $81 \%$ of the population under the age of 40 [19]. GBD 2010 reported that life expectancy has increased from 72.5 and 76.3 years in 1990 to 75.0 and 79.9 years in 2010 for men and women, respectively [20]. As life expectancy increases and the size of population is on the rise, the burden of hypertension and other chronic diseases, if uncontrolled, will pose major challenges to the health system. Prevention should be a priority for all in KSA. As the KSA MOH has successfully tackled the burden of infectious diseases through prevention, the same effort should be applied to reduce the burden of chronic diseases.

\section{Conflict of Interests}

The authors declare that there is no conflict of interests regarding the publication of this paper. 
TABLE 4: Multivariate logistic regression for sociodemographic characteristics, risk factors, and borderline hypertension, in Saudi Arabia, of males and females aged 15 years or older, 2013.

\begin{tabular}{|c|c|c|c|c|c|}
\hline \multirow{2}{*}{ Sociodemographic and risk factors } & \multirow{2}{*}{ Categories } & \multicolumn{2}{|c|}{ Sociodemographic model } & \multicolumn{2}{|c|}{ Full adjusted model } \\
\hline & & AOR & $95 \% \mathrm{CI}$ & AOR & $95 \% \mathrm{CI}$ \\
\hline \multirow{2}{*}{ Sex } & Males & REF & & REF & \\
\hline & Females & 0.44 & $0.38-0.50$ & 0.36 & $0.31-0.43$ \\
\hline Age* $^{*}$ & & 1.04 & $1.03-1.05$ & 1.03 & $1.02-1.04$ \\
\hline \multirow{3}{*}{ Marital status } & Currently married & REF & & REF & \\
\hline & Never married & 0.79 & $0.66-0.94$ & 0.80 & $0.65-0.99$ \\
\hline & Separated, divorced, or widowed & 1.15 & $0.84-1.58$ & 1.39 & $0.93-2.06$ \\
\hline \multirow{3}{*}{ Education } & Primary school or less & REF & & & \\
\hline & Elementary or high school completed & 0.95 & $0.80-1.14$ & & \\
\hline & College degree or higher education & 1.04 & $0.85-1.26$ & & \\
\hline \multirow{4}{*}{$\begin{array}{l}\text { Daily servings of red meat and } \\
\text { chicken }\end{array}$} & $0-1$ & & & REF & \\
\hline & $1-2$ & & & 0.95 & $0.79-1.14$ \\
\hline & $2-3$ & & & 0.69 & $0.54-0.88$ \\
\hline & $3+$ & & & 0.95 & $0.76-1.19$ \\
\hline \multirow{4}{*}{ Daily hours spent watching TV } & $0-1$ & & & REF & \\
\hline & $1-3$ & & & 1.03 & $0.75-1.42$ \\
\hline & $3-5$ & & & 1.25 & $0.90-1.74$ \\
\hline & $5+$ & & & 1.20 & $0.85-1.69$ \\
\hline \multirow{4}{*}{ Levels of physical activity } & None & & & REF & \\
\hline & Low & & & 0.99 & $0.81-1.21$ \\
\hline & Moderate & & & 0.77 & $0.60-0.98$ \\
\hline & High & & & 0.87 & $0.70-1.06$ \\
\hline \multirow{2}{*}{ Obesity } & Not obese & & & REF & \\
\hline & Obese & & & 1.55 & $1.30-1.83$ \\
\hline \multirow{2}{*}{ History of diagnosis with diabetes } & No & & & REF & \\
\hline & Yes & & & 1.16 & $0.83-1.63$ \\
\hline \multirow{2}{*}{ Diagnosis of chronic condition } & No & & & REF & \\
\hline & Yes & & & 0.89 & $0.64-1.24$ \\
\hline
\end{tabular}

${ }^{*}$ AOR for age should be considered as for an increase of one year.

Odds ratios were adjusted for sociodemographic characteristics: sex, age, marital status, and education.

AOR: adjusted odds ratios; CI: confidence intervals; REF: reference.

TABLE 5: Multivariate logistic regression for sociodemographic characteristics, risk factors, and undiagnosed hypertension, in Saudi Arabia, of males and females aged 15 years or older, 2013.

\begin{tabular}{|c|c|c|c|c|c|}
\hline \multirow{2}{*}{ Sociodemographic and risk factors } & \multirow{2}{*}{ Categories } & \multicolumn{2}{|c|}{ Sociodemographic model } & \multicolumn{2}{|c|}{ Full adjusted model } \\
\hline & & $\mathrm{AOR}$ & $95 \% \mathrm{CI}$ & AOR & $95 \% \mathrm{CI}$ \\
\hline \multirow{2}{*}{ Sex } & Males & REF & & REF & \\
\hline & Females & 0.54 & $0.44-0.66$ & 0.54 & $0.44-0.67$ \\
\hline Age $^{*}$ & Age & 1.06 & $1.05-1.06$ & 1.05 & 1.05-1.06 \\
\hline \multirow{3}{*}{ Marital status } & Currently married & REF & & & \\
\hline & Never married & 0.77 & $0.57-1.03$ & 0.78 & $0.58-1.05$ \\
\hline & Separated, divorced, or widowed & 1.17 & $0.85-1.61$ & 1.13 & $0.81-1.57$ \\
\hline \multirow{3}{*}{ Education } & Primary school or less & REF & & & \\
\hline & Elementary or high school completed & 0.96 & $0.75-1.23$ & 0.97 & $0.76-1.25$ \\
\hline & College degree or higher education & 0.77 & $0.59-1.02$ & 0.79 & $0.60-1.03$ \\
\hline \multirow{2}{*}{$\begin{array}{l}\text { History of diagnosis with } \\
\text { prediabetes }\end{array}$} & No & & & REF & \\
\hline & Yes & & & 0.73 & $0.41-1.31$ \\
\hline \multirow{2}{*}{ History of diagnosis with diabetes } & No & & & REF & \\
\hline & Yes & & & 1.46 & 1.09-1.94 \\
\hline
\end{tabular}

*AOR for age should be considered as for an increase of one year.

Odds ratios were adjusted for sociodemographic characteristics: sex, age, marital status, and education.

AOR: adjusted odds ratios; CI: confidence intervals; REF: reference. 


\section{Acknowledgments}

The authors would like to acknowledge Kate Muller at the Institute for Health Metrics and Evaluation, Seattle, WA, for editing this paper. The salaries of the authors from the Institute for Health Metrics and Evaluation (IHME) were fully or partially paid through a grant from the Ministry of Health $(\mathrm{MOH})$ of the Kingdom of Saudi Arabia. The MOH managed the data collection for this study.

\section{References}

[1] H. Wang, L. Dwyer-Lindgren, K. T. Lofgren et al., "Age-specific and sex-specific mortality in 187 countries, 1970-2010: a systematic analysis for the Global Burden of Disease study 2010," The Lancet, vol. 380, no. 9859, pp. 2071-2094, 2012.

[2] F. H. Messerli, B. Williams, and E. Ritz, "Essential hypertension," The Lancet, vol. 370, no. 9587, pp. 591-603, 2007.

[3] T. K. Singh, V. Arya, and N. Navaratnarajah, "Chronic kidney disease and cardiovascular disease: a focus on primary care," Cardiovascular \& Hematological Disorders-Drug Targets, 2014.

[4] J. He and P. K. Whelton, "Elevated systolic blood pressure as a risk factor for cardiovascular and renal disease," Journal of Hypertension, vol. 17, no. 2, pp. S7-S13, 1999.

[5] W. J. Elliott, "The economic impact of hypertension," The Journal of Clinical Hypertension, vol. 5, no. 3, supplement 2, pp. 3-13, 2003.

[6] P. M. Kearney, M. Whelton, K. Reynolds, P. Muntner, P. K. Whelton, and J. He, "Global burden of hypertension: analysis of worldwide data," The Lancet, vol. 365, no. 9455, pp. 217-223, 2005.

[7] Institute for Health Metrics and Evaluation (IHME), "GBD arrow Diagram, Saudi Arabia. Risk of deaths.1990-2010," IHME, University of Washington, Seattle, Wash, USA, 2013, http://www.healthmetricsandevaluation.org/gbd/visualizations/ gbd-arrow-diagram.

[8] Institute for Health Metrics and Evaluation (IHME), Stacked bar chart, Saudi Arabia. Deaths.1990-2010 [Internet], IHME, University of Washington, Seattle, Wash, USA, 2013, http://viz .healthmetricsandevaluation.org/gbd-compare/.

[9] M. M. Al-Nozha, M. Abdullah, M. R. Arafah et al., "Hypertension in Saudi Arabia," Saudi Medical Journal, vol. 28, no. 1, pp. 77-84, 2007.

[10] N. Al-Hamdan, A. Saeed, A. Kutbi, A. J. Choudhry, and R. Nooh, "Characteristics, risk factors, and treatment practices of known adult hypertensive patients in Saudi Arabia," International Journal of Hypertension, vol. 2010, Article ID 168739, 7 pages, 2010.

[11] A. A. Saeed, N. A. Al-Hamdan, A. A. Bahnassy, A. M. Abdalla, M. A. F. Abbas, and L. Z. Abuzaid, "Prevalence, awareness, treatment, and control of hypertension among Saudi adult population: a national survey," International Journal of Hypertension, vol. 2011, Article ID 174135, 8 pages, 2011.

[12] C. L. Craig, A. L. Marshall, M. Sjöström et al., "International physical activity questionnaire: 12 -country reliability and validity," Medicine and Science in Sports and Exercise, vol. 35, no. 8, pp. 1381-1395, 2003.

[13] National Heatlth and Nutrition Examination Survey (NHANES), Health Tech/Blodd Pressure Procedures Manual. [Internet], CDC, 2009, http://www.cdc.gov/nchs/data/nhanes/ nhanes_09_10/BP.pdf.
[14] F. R. M. Causland, F. M. Sacks, and J. P. Forman, "Marital status, dipping and nocturnal blood pressure: results from the dietary approaches to stop hypertension trial," Journal of Hypertension, vol. 32, no. 4, pp. 756-761, 2014.

[15] A. Lipowicz and M. Lopuszanska, "Marital differences in blood pressure and the risk of hypertension among Polish men," European Journal of Epidemiology, vol. 20, no. 5, pp. 421-427, 2005.

[16] H. Alwan, M. Pruijm, B. Ponte et al., "Epidemiology of masked and white-coat hypertension: the family-based SKIPOGH study," PloS ONE, vol. 9, no. 3, Article ID e92522, 2014.

[17] "Race, education and prevalence of hypertension," American Journal of Epidemiology, vol. 106, no. 5, pp. 351-361, 1977.

[18] F. D. Fuchs, L. B. Moreira, R. S. Moraes, M. Bredemeier, and S. C. Cardozo, "Prevalence of systemic arterial hypertension and associated risk factors in the Porto Alegre metropolitan area. Populational-based study," Arquivos Brasileiros de Cardiologia, vol. 63, no. 6, pp. 473-479, 1994.

[19] Ministry of Health, Kingdom of Saudi Arabia Projected Population, 2013.

[20] Institute for Health Metrics and Evaluation (IHME), Healthy Years Lost vs Life Expectancy, Saudi Arabia. Risk of Deaths. 1990-2010, University of Washington, Seattle, Wash, USA, 2013, http://www.healthmetricsandevaluation.org/gbd/visualizations/ country. 


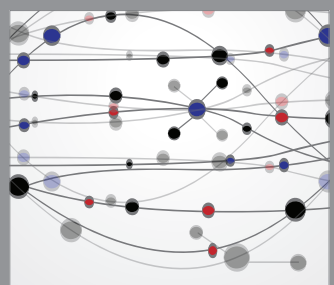

The Scientific World Journal
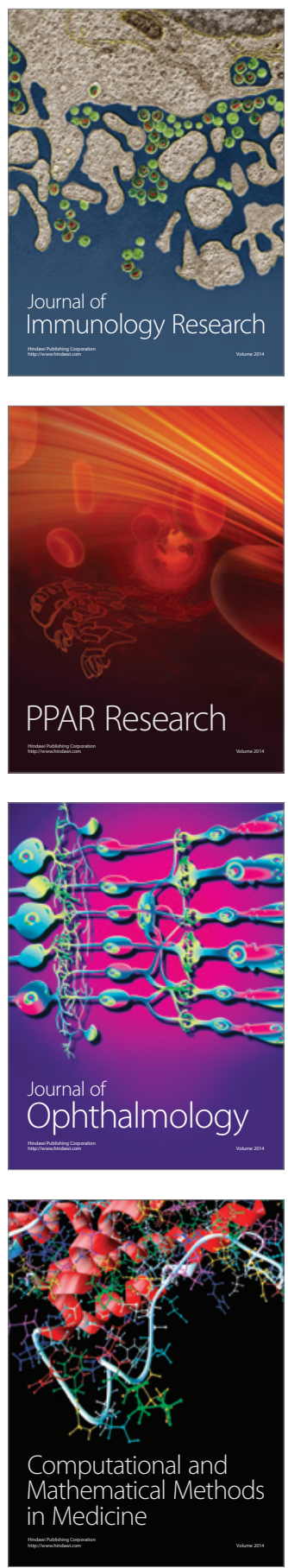

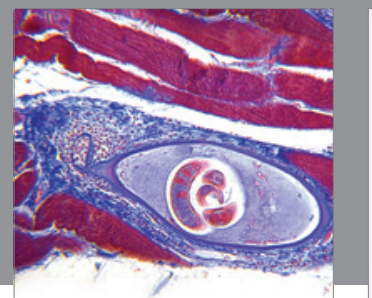

Gastroenterology

Research and Practice
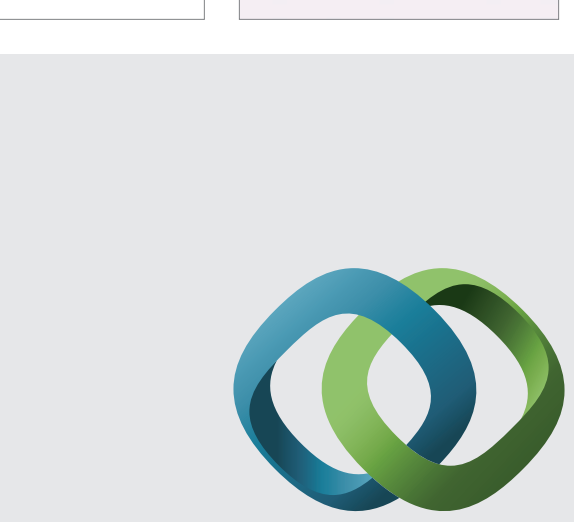

\section{Hindawi}

Submit your manuscripts at

http://www.hindawi.com
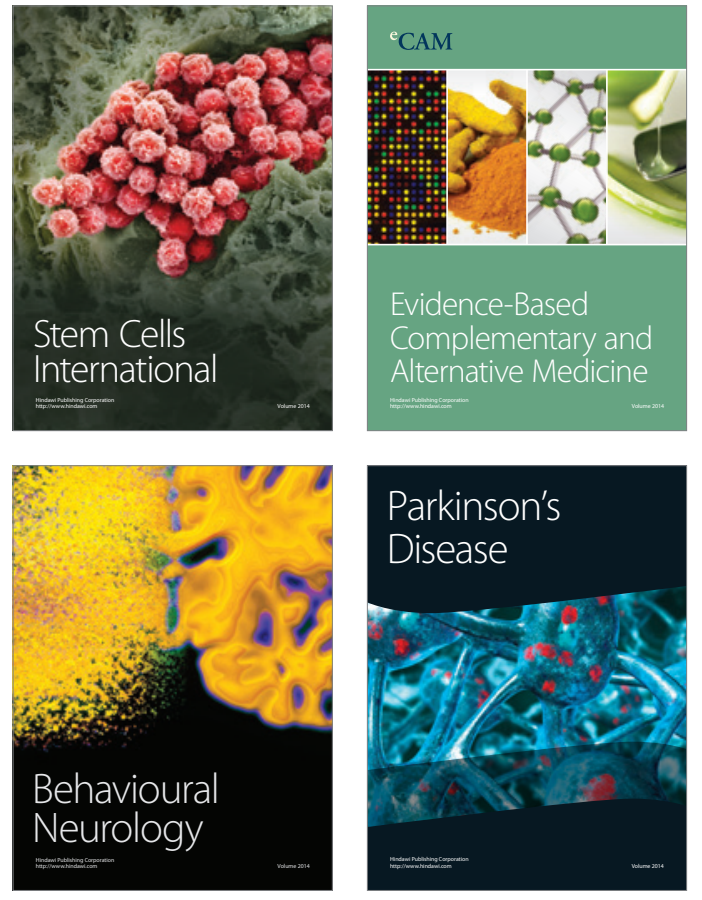
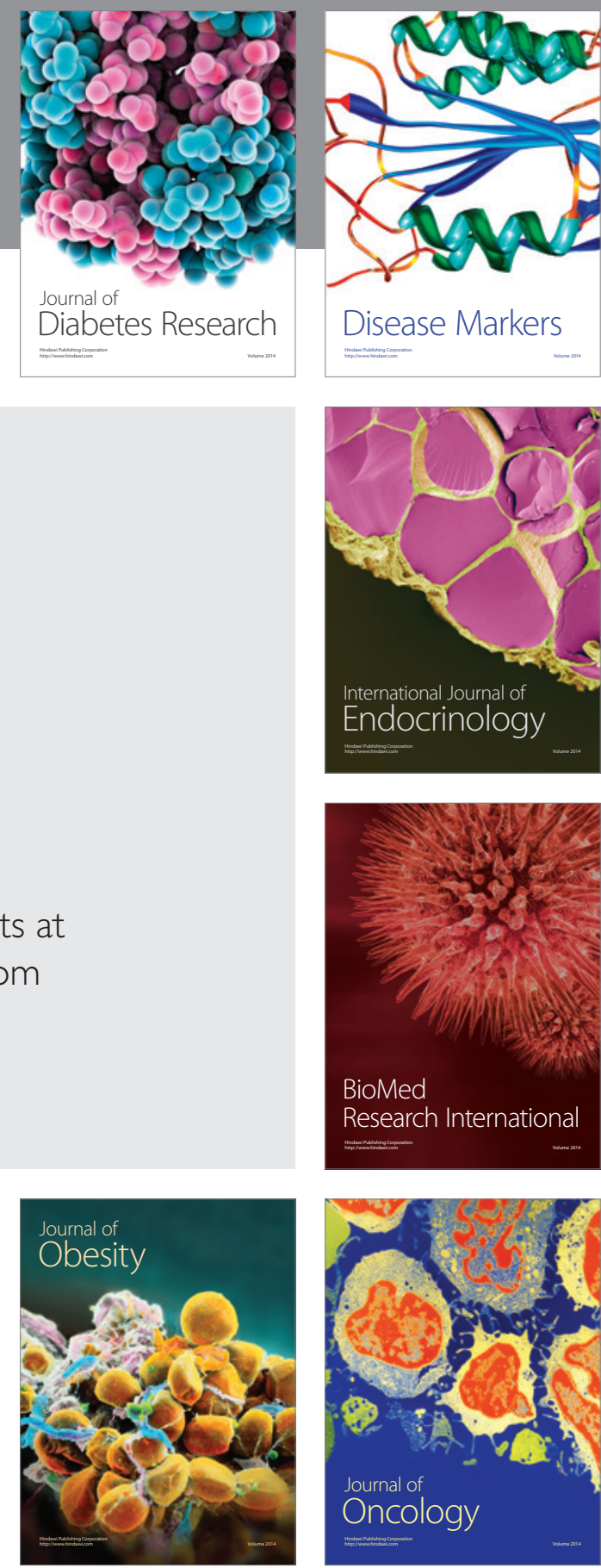

Disease Markers
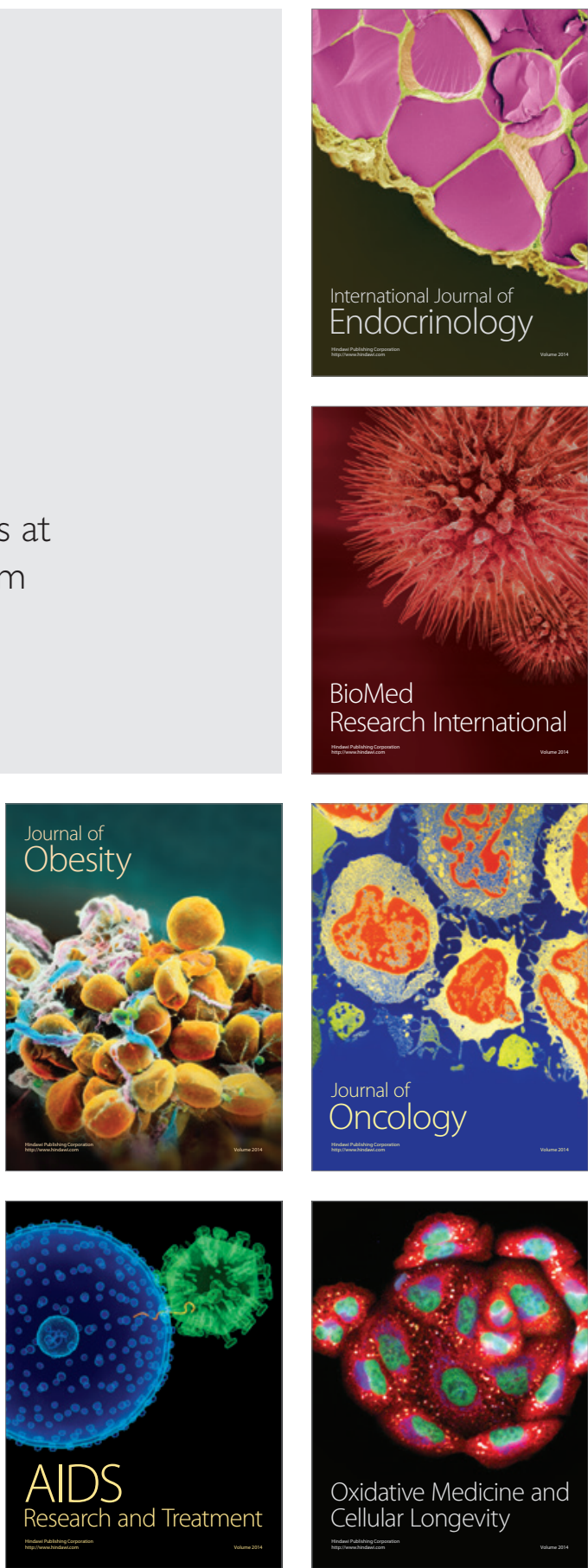